\title{
On to Meaningful Use Stage 2: Refining Public Health Readiness Guidelines
}

\author{
Sanjeev Tandon*1 and Charlie Ishikawa ${ }^{2}$ \\ ${ }^{1}$ Centers for Disease Control \& Prevention (CDC), Atlanta, GA, USA; ' International Society for Disease Surveillance (ISDS), Boston, \\ MA, USA
}

\section{Objective}

To exchange lessons learned and refine national guidelines for public health agencies to declare Meaningful Use readiness, register eligible professionals and hospitals for the public health meaningful use objectives, on-board data providers, and perform "on-going" data submission.

\section{Introduction}

In the U.S., federal programs are accelerating the meaningful use of electronic health record (EHR) technology and encouraging greater standardization in how governmental public health agencies (PHAs) establish surveillance data partnerships with healthcare providers. To qualify for the benefits of these federal programs (a.k.a., Meaningful Use), healthcare professionals and hospitals must determine: 1) Whether their jurisdictional PHA collects health data for immunization or cancer registries, reportable diseases, and/or syndromic surveillance; and 2) If the PHA does collect this data, then they must register for data on-boarding with the PHA and actively work with them to establish on-going data exchange. These requirements are predicated on participating state and local PHAs either establishing new or expanding the capacity of their existing public health data reporting services. To assist state and local PHAs in this effort, the U.S. Centers for Disease Control and Prevention (CDC) facilitates a national task force, known as the Stage 2 MU Public Health Reporting Requirements Task Force, which has recommended guidelines and clarified requirements for these new processes.

\section{Description}

During this Round Table discussion, participants will exchange lessons learned in implementing Meaningful Use to refine the national guidelines developed by the CDC's Meaningful Use Stage 2 Public Health Reporting Requirements Task Force to aid governmental public health agencies in forming data sharing partnerships. The discussion will highlight areas where these guidelines have been effective and broadly adopted, as well as, identify key areas for improvements.

\section{Audience Engagement}

To facilitate discussion and collaborative learning, we will engage the audience to provide input on lessons learned and best practices regarding:

1. Eligible professionals (EPs) and hospitals (EHs) accessing their PHA's Meaningful Use readiness information through a national database

2. Registering the intent of EPs and EHs for Meaningful Use data on-boarding

3. Working with EPs and EHs to on-board their data under the new requirements for responsiveness

4. Providing acknowledgments of on-going data provision

After a brief review of the guidelines with the audience, they will be presented with the following 3 questions to help focus the conversation:

1. In what ways has your agency done something similar or different than the MU Taskforce guidelines?
2. From your experience, what is missing in the guidelines?

3. In what ways are these Meaningful Use processes helping and hindering your agency's efforts to improve disease surveillance capabilities?

\section{Conclusions}

We will produce a list of lessons learned first in small focus groups of no more than five people, and then synthesize the small group work into a large list of lessons learned and best practices, which will be disseminated more broadly to the surveillance community.

\section{Keywords}

Public Health; Electrionic Health Records; Meaningful Use

\section{Acknowledgments}

Stage 2 Meaningful Use Public Health Reporting Requirements Task Force

\author{
*Sanjeev Tandon \\ E-mail: STandon@cdc.gov
}

